\title{
Randomly poled crystals as a source of photon pairs
}

\author{
Jan Peřina Jr. ${ }^{1}$ and Jiří Svozilík ${ }^{1}$ \\ ${ }^{1}$ Joint Laboratory of Optics, Palacký University and Institute of Physics of Academy of Sciences of the Czech Republic, \\ 17. listopadu 50a, 772 07 Olomouc, Czech Republif*
}

\begin{abstract}
Generation of photon pairs from randomly poled nonlinear crystals is investigated using analytically soluble model and numerical calculations. Randomly poled crystals are discovered as sources of entangled ultra broad-band signal and idler fields. Their photon-pair generation rates scale linearly with the number of domains. Entanglement times as short as several fs can be reached. Comparison with chirped periodically-poled structures is given and reveals close similarity.
\end{abstract}

PACS numbers: 42.65.Lm, 42.50.Dv, 46.65.+g

\section{INTRODUCTION}

The first experimentally observed nonlinear optical effect, second-harmonic generation, was investigated already more than forty years ago by Franken [1]. Since that, many other nonlinear effects have been revealed and understood even at quantum level. Among them, spontaneous parametric down-conversion (SPDC) with its production of photon pairs belongs to the most fascinating. The reason is that two photons comprising a photon pair generated in one quantum event of this process are mutually strongly correlated (entangled) as was discovered by Hong, $\mathrm{Ou}$ and Mandel in eighties [2]. They used in their experiments nonlinear bulk crystals that later become the most common sources of photon pairs [3]. In order to observe spontaneous parametric down-conversion phase-matching conditions of the interacting three optical fields have to be fulfilled. Unfortunately, they cannot be naturally fulfilled in many highly nonlinear crystals. However, Armstrong [4] has arrived with the concept of additional periodic modulation of nonlinear susceptibility that has been practically developed in periodical poling of nonlinear crystals [5]. In this concept, wave vector of the additionally introduced nonlinear modulation is added to the natural phase-matching condition and the so-called quasi-phase-matching of the interacting optical fields is reached this way. Highly nonlinear materials can be exploited since then. We note that shortening of a nonlinear medium such that phase-matching conditions loose their importance is the only alternative way to periodical poling. This approach has been applied in photonic-bandgap structures in which optical interference is crucial for reaching an efficient nonlinear interaction 6-11].

Periodical poling has occurred to be extraordinarily useful. It has provided not only compensation for the natural phase mismatch. The ability to tailor the properties of emitted optical fields has been revealed soon. It is based on using ordered nonlinear domains with variable lengths (chirped periodical poling). Presence of domains of different lengths in an ordered structure allows an efficient nonlinear interaction of fields in a broad spectral

\footnotetext{
* perinaj@prfnw.upol.cz
}

range. For example, signal and idler fields with spectra several hundreds of $\mathrm{nm}$ wide can be generated in chirped $\mathrm{LiNbO}_{3}$ crystals. On the other hand, domains with different lengths can also be ordered randomly. A bit surprisingly, the nonlinear interaction can be efficient even in this case sometimes referred as stochastic quasi-phasematching (SQPM). Similarly as ordered poled structures the randomly poled structures (RPS) allow spectrally broadband nonlinear interaction. It is not surprising that efficiency of random structures is worse compared to ordered ones. However, they usually put smaller requirements to polarization properties of the interacting optical fields as well as orientation of the nonlinear medium [12]. Also fabrication of RPS is much easier because high precision is required in production of ordered periodicallypoled structures.

The role of SQPM in 1D has already been addressed for the process of second-harmonic generation [13 17] and the process of difference-frequency generation [18]. Moreover full domain random structures allowing SQPM for transversal second-harmonic generation have been studied in [19].

Here, we focus our attention to the generation of photon pairs in randomly poled 1D nonlinear crystals [20]. It is shown that spectral properties of photon pairs and photon-pair generation rates are comparable in RPS and chirped periodically-poled structures (CPPS) [21 24]. This is very promising for easy production of broadband and efficient sources of photon pairs. These broadband sources are important, e.g., in metrology (quantum optical coherence tomography 25]) or quantum-information processing [26, 27]. We note that broadband photon-pair sources can also be constructed using zero group-velocity dispersion conditions 28]. However, such conditions can be met only for certain pump frequencies considering a given material.

The paper is organized as follows. In Sec. II, a general theory of SPDC modified to random structures is presented. Photon-pair generation rates and intensity spectra are studied both for random and chirped structures in Sec. III. Sec. IV is devoted to temporal properties of the generated photon pairs. Spatial properties of photon pairs are addressed in Sec. V. Temperature behavior of the quantities characterizing photon pairs is analyzed 
in Sec. VI. Sec. VII brings the analysis of fabrication errors. The role of ordering in chirped structures is studied in Sec. VIII. Finally, conclusions are drawn in Sec. IX.

\section{SPONTANEOUS PARAMETRIC DOWN-CONVERSION IN POLED NONLINEAR CRYSTALS}

The process of SPDC in a nonlinear crystal can be conveniently described by the following interaction Hamiltonian $\hat{H}_{\text {int }}[29,30]$ :

$$
\begin{aligned}
& \hat{H}_{\mathrm{int}}(t)=\varepsilon_{0} \mathcal{B} \int_{-L}^{0} d z \chi^{(2)}(z) \\
& \times E_{p}^{(+)}(z, t) \hat{E}_{s}^{(-)}(z, t) \hat{E}_{i}^{(-)}(z, t)+\text { H.c. }
\end{aligned}
$$

$L$ denotes the crystal length. In Eq. (10), the positivefrequency part of the pump electric-field amplitude is denoted as $\mathbf{E}_{p}^{(+)}$and $\mathbf{E}_{s}^{(-)}\left(\mathbf{E}_{i}^{(-)}\right)$stands for the negativefrequency part of the signal (idler) electric-field amplitude operator. The $z$-dependent second-order susceptibility tensor $\chi^{(2)}$ describes poling of the nonlinear material. Vacuum permittivity is denoted as $\varepsilon_{0}$ and $\mathcal{B}$ means the transverse area of the optical fields. Symbol H.c. replaces the Hermitian-conjugated term.

Electric-field amplitudes occurring in Eq. (1) can be conveniently decomposed into harmonic plane waves with frequencies $\omega_{a}$ and wave vectors $k_{a}$ :

$$
\begin{gathered}
\hat{E}_{a}^{(-)}(z, t)=\frac{1}{2 \pi} \int d \omega_{a} \hat{E}_{a}^{(-)}\left(\omega_{a}\right) \exp \left(-i k_{a} z+i \omega_{a} t\right), \\
a=s, i .
\end{gathered}
$$

The quantum spectral amplitudes $\hat{E}_{a}^{(-)}\left(\omega_{a}\right)$ in Eq. (2) can then be expressed using photon creation operators $\hat{a}_{a}^{\dagger}\left(\omega_{a}\right)$ :

$$
\hat{E}_{a}^{(-)}\left(\omega_{a}\right)=-i \sqrt{\frac{\hbar \omega_{a}}{2 \varepsilon_{0} c n_{a}\left(\omega_{a}\right) \mathcal{B}}} \hat{a}_{a}^{\dagger}\left(\omega_{a}\right) ;
$$

$c$ is speed of light in vacuum, $\hbar$ reduced Planck constant, and $n_{a}$ stands for index of refraction of field $a$.

First-order perturbation solution of Schrödinger equation with the initial vacuum state $|\mathrm{vac}\rangle$ in the signal and idler fields results in the following two-photon quantum state $|\psi\rangle$ :

$$
|\psi\rangle=\int d \omega_{s} \int d \omega_{i} \Phi\left(\omega_{s}, \omega_{i}\right) \hat{a}_{s}^{\dagger}\left(\omega_{s}\right) \hat{a}_{i}^{\dagger}\left(\omega_{i}\right)|\mathrm{vac}\rangle .
$$

The two-photon spectral amplitude $\Phi$ introduced in Eq. (44) is given as follows:

$$
\begin{aligned}
\Phi\left(\omega_{s}, \omega_{i}\right)= & g\left(\omega_{s}, \omega_{i}\right) E_{p}^{(+)}\left(\omega_{s}+\omega_{i}\right) \\
& \times F\left(\Delta k\left(\omega_{s}, \omega_{i}\right)\right),
\end{aligned}
$$

where $g$ denotes a coupling constant, $g\left(\omega_{s}, \omega_{i}\right)=$ $i \sqrt{\omega_{s} \omega_{i}} /\left[2 c \pi \sqrt{n_{s}\left(\omega_{s}\right) n_{i}\left(\omega_{i}\right)}\right] \chi^{(2)}(0)$, and $E_{p}^{(+)}\left(\omega_{p}\right)$ stands for the pump-field amplitude spectrum. The stochastic function $F$ introduced in Eq. (5) describes SQPM and is derived in the form [20]:

$$
F(\Delta k)=\int_{-L}^{0} d z \frac{\chi^{(2)}(z)}{\chi^{(2)}(0)} \exp (i \Delta k z) .
$$

Symbol $\Delta k$ describes the natural phase mismatch for the interacting fields, $\Delta k=k_{p}-k_{s}-k_{i}$.

In a periodically-poled structure neighbor domains differ in signs of $\chi^{(2)}$ nonlinearity and function $F$ defined in Eq. (6) can be recast into the form:

$$
F(\Delta k)=\sum_{n=1}^{N_{L}}(-1)^{n-1} \int_{z_{n-1}}^{z_{n}} d z \exp (i \Delta k z) .
$$

Symbol $N_{L}$ denotes the number of domains and $n$-th domain extends from $z=z_{n-1}$ to $z=z_{n}$. Positions $z_{n}$ of domain boundaries are random and can be expressed as $z_{n}=z_{n-1}+l_{0}+\delta l_{n}\left(n=1, \ldots, N_{L}, z_{0}=-L\right)$ in our model using stochastic Gaussian declinations $\delta l_{n}$. The basic layer length $l_{0}$ is determined such that quasi-phasematching is reached, i.e. $l_{0}=\pi / \Delta k_{0}, \Delta k_{0} \equiv \Delta k\left(\omega_{s}^{0}, \omega_{i}^{0}\right)$, and $\omega_{a}^{0}$ means central frequency of field $a$. The random declinations $\delta l_{n}$ are mutually independent and can be described by the joint Gaussian probability distribution $P$ :

$$
P(\delta \mathbf{L})=\frac{1}{(\sqrt{\pi} \sigma)^{N_{L}}} \exp \left(-\delta \mathbf{L}^{T} \mathbf{B} \delta \mathbf{L}\right) .
$$

Covariance matrix $\mathbf{B}$ is assumed to be diagonal and its nonzero elements equal $1 / \sigma^{2}$. Stochastic vector $\delta \mathbf{L}$ is composed of declinations $\delta l_{n}$; symbol ${ }^{T}$ stands for transposition. Characteristic function $G$ of the distribution $P$ in Eq. (8) takes the form:

$$
G(\delta \mathbf{K}) \equiv\langle\exp (i \delta \mathbf{K} \cdot \delta \mathbf{L})\rangle_{\mathrm{av}}=\prod_{j=1}^{N} G\left(\delta k_{j}\right) ;
$$

symbol - means scalar product. Vector $\delta \mathbf{K}$ of parameters of the characteristic function $G$ is composed of elements $\delta k_{j}$. One-dimensional characteristic function $G(\delta k)$ in Eq. (9) equals $\exp \left(-\sigma^{2} \delta k^{2} / 4\right)$.

In order to obtain analytical formulas, we integrate the expression for function $F$ in Eq. (7) domain by domain and modify the contributions of the first and last domains in such a way that the following simple formula is reached:

$$
F(\Delta k)=\frac{2 i}{\Delta k} \sum_{n=0}^{N_{L}}(-1)^{n} \exp \left(i \Delta k z_{n}\right) .
$$

As a typical structure contains hundreds of domains, incorrect inclusion of fields from the first and the last domains leads to negligible declinations. The formula in Eq. (10) can be interpreted such that SPDC occurs only in domains with positive susceptibility $\chi^{(2)}$ at doubled 
amplitude and domains with negative susceptibility $\chi^{(2)}$ play only the role of a 'linear' filler. This interpretation elucidates why a pair of domains having one positive and one negative signs of susceptibility $\chi^{(2)}$ forms an elementary unit for understanding properties of photon pairs.

\section{PHOTON-PAIR GENERATION RATES AND INTENSITY SPECTRA}

Photon-pair generation rate as well as intensity spectra can be easily derived from mean spectral density of the number of generated photon pairs $n\left(\omega_{s}, \omega_{i}\right)$. The mean spectral density $n$ corresponding to the quantum state $|\psi\rangle$ is defined by the formula

$$
n\left(\omega_{s}, \omega_{i}\right)=\left\langle\left\langle\psi\left|\hat{a}_{s}^{\dagger}\left(\omega_{s}\right) \hat{a}_{s}\left(\omega_{s}\right) \hat{a}_{i}^{\dagger}\left(\omega_{i}\right) \hat{a}_{i}\left(\omega_{i}\right)\right| \psi\right\rangle\right\rangle_{\mathrm{av}},
$$

where the symbol \langle\rangle$_{\text {av }}$ means stochastic averaging over an ensemble of geometric configurations of an RPS. Assuming the quantum state $|\psi\rangle$ written in Eq. (4) we arrive at the formula:

$$
\begin{aligned}
n\left(\omega_{s}, \omega_{i}\right)= & \left|g\left(\omega_{s}, \omega_{i}\right)\right|^{2}\left|E_{p}^{(+)}\left(\omega_{s}+\omega_{i}\right)\right|^{2} \\
& \times\left\langle\left|F\left(\Delta k\left(\omega_{s}, \omega_{i}\right)\right)\right|^{2}\right\rangle_{\mathrm{av}} .
\end{aligned}
$$

Spectrum $S_{s}$ of, e.g., the signal field and photon-pair generation rate $N$ can then be derived using the expressions:

$$
\begin{aligned}
& S_{s}=\hbar \omega_{s} \int d \omega_{i} n\left(\omega_{s}, \omega_{i}\right), \\
& N=\int d \omega_{s} \int d \omega_{i} n\left(\omega_{s}, \omega_{i}\right) .
\end{aligned}
$$

The averaged squared modulus of the phase-matching function $F$ as determined by the formula in Eq. (10) can be written in the form:

$$
\begin{gathered}
\left\langle|F(\Delta k)|^{2}\right\rangle_{\text {av }}=\frac{4}{\Delta k^{2}}\left(\left(N_{L}+1\right) \frac{1-|H(\delta k)|^{2}}{|1-H(\delta k)|^{2}}\right. \\
\left.-\left[\frac{H(\delta k)\left[1-H(\delta k)^{N_{L}+1}\right]}{[1-H(\delta k)]^{2}}+\text { c.c. }\right]\right),
\end{gathered}
$$

$\delta k\left(\omega_{s}, \omega_{i}\right)=\Delta k\left(\omega_{s}, \omega_{i}\right)-\Delta k_{0}$. Symbol c.c. replaces the complex-conjugated term. Function $H(\delta k)$ occurring in Eq. (15) is defined as:

$$
\begin{aligned}
H(\delta k) & =\exp \left[i \delta k l_{0}\right] G\left(\Delta k_{0}+\delta k\right), \\
G(\Delta k) & =\exp \left(-\frac{\sigma^{2} \Delta k^{2}}{4}\right) .
\end{aligned}
$$

The averaged squared modulus $\left\langle|F(\Delta k)|^{2}\right\rangle_{\text {av }}$ of phasematching function given in Eq. (15) determines the averaged spectral density $n$ and behaves as follows. It holds that $|H| \leq 1$ and $|H|=1$ for a fully ordered structure. If $\delta k=0$ in a fully ordered structure, $H$ is real $(H=1)$ and the averaged squared phase-matching function $\left\langle|F(\Delta k)|^{2}\right\rangle_{\text {av }}$ reaches its maximum value $4\left(N_{L}+1\right)^{2}$. Nonzero phase mismatch $\delta k$ shifts $H$ into the complex plane which results in lower values of the mean value
$\left\langle|F(\Delta k)|^{2}\right\rangle_{\text {av }}$. The larger the $\delta k$, the smaller the mean value $\left\langle|F(\Delta k)|^{2}\right\rangle_{\mathrm{av}}$. Inspection of the formula for $H$ in Eq. (16) also shows that the larger the value of the basic layer length $l_{0}$ the faster the decrease of mean values $\left\langle|F(\Delta k)|^{2}\right\rangle_{\text {av }}$ for given $\delta k$. According to the formula in Eq. (16) the larger the standard deviation $\sigma$ of a random structure the smaller the value of $|H|$. The decrease of values of $|H|$ results in an increase of the range of values of the phase mismatch $\delta k$ in which the averaged squared modulus $\left\langle|F(\Delta k)|^{2}\right\rangle_{\text {av }}$ of phase-matching function attains non-negligible values [see the formula in Eq. (15)].

The formula for averaged squared modulus $\left\langle|F(\Delta k)|^{2}\right\rangle_{\text {av }}$ of phase-matching function in Eq. (15) can be substantially simplified under the assumption $\sigma^{2}\left(\Delta k_{0}\right)^{2} N_{L} / 2 \gg 1$ :

$$
\begin{gathered}
\left\langle|F(\Delta k)|^{2}\right\rangle_{\text {av }}=\frac{2 N_{L}}{\left(\Delta k_{0}+\delta k\right)^{2}} \\
\times \frac{1-G\left(\Delta k_{0}+\delta k\right)}{1-2 G\left(\Delta k_{0}+\delta k\right) \cos \left(\delta k l_{0}\right)+G\left(\Delta k_{0}+\delta k\right)^{2}} .
\end{gathered}
$$

Increasing values of phase mismatch $\delta k$ lead to greater values of the denominator in the fraction on the r.h.s. of Eq. (17) that result in the decrease of values of the averaged squared modulus $\left\langle|F(\Delta k)|^{2}\right\rangle_{\text {av }}$ of phase-matching function. On the other hand, increasing values of deviation $\sigma$ weaken this behavior.

For comparison, we consider another type of RPS defined such that $z_{n}=-L+n l_{0}+\delta l_{n}$ where $\delta l_{n}$ is a random declination of the $n$-th boundary. These 'weaklyrandom' structures are more ordered compared to those considered earlier because the change of length of an $n$ th domain is compensated by the change in length of an $(n+1)$-th domain. The averaged squared modulus $\left\langle\left|F^{\mathrm{w}-\mathrm{r}}(\Delta k)\right|^{2}\right\rangle_{\text {av }}$ of phase-matching function can be derived in this case as follows:

$$
\begin{aligned}
& \left\langle\left|F^{\mathrm{w}-\mathrm{r}}(\Delta k)\right|^{2}\right\rangle_{\mathrm{av}}=\frac{4}{(\Delta k)^{2}}\left\{N_{L}+1\right. \\
& +\left|G\left(\Delta k_{0}+\delta k\right)\right|^{2}\left[\frac{\exp \left(i \delta k l_{0}\right)}{1-\exp \left(i \delta k l_{0}\right)}\right. \\
& \left.\left.\times\left(N_{L}-\exp \left(i \delta k l_{0}\right) \frac{1-\exp \left[i \delta k l_{0} N_{L}\right]}{1-\exp \left(i \delta k l_{0}\right)}\right)+\text { c.c. }\right]\right\} .
\end{aligned}
$$

Disorder of the boundary positions manifests itself as a filter for the averaged squared modulus $\left\langle\left|F^{\mathrm{w}-\mathrm{r}}(\Delta k)\right|^{2}\right\rangle_{\text {av }}$ of phase-matching function, as evident from the expression in Eq. (18). This leads to spectral filtering of the spectral density $n$. This behavior is qualitatively different from that observed in RPS as described by the formula in Eq. (15) indicating broadening of the spectral density $n$ with increasing values of the deviation $\sigma$.

Spectral broadening is the most interesting feature of ordered CPPS that we consider here for comparison. Positions of boundaries in these structures are described by 
the formula $z_{n}=-L+n l_{0}+\zeta^{\prime}\left(n-N_{L} / 2\right)^{2} l_{0}^{2}, \zeta^{\prime}=\zeta / \Delta k_{0}$, and $\zeta$ denotes chirping parameter. The phase-matching function $F^{\text {chirp }}(\Delta k)$ then takes the form [22]:

$$
\begin{aligned}
& F^{\operatorname{chirp}}(\Delta k)=\frac{2 \sqrt{\pi}}{\sqrt{i \Delta k^{3} \zeta^{\prime}} l_{0}} \exp \left(i \Delta k N_{L} l_{0} / 2\right) \\
& \times \exp \left(-\frac{i \delta k^{2}}{4 \Delta k \zeta^{\prime}}\right)\left[\operatorname{erf}\left(f\left(N_{L} / 2\right)\right)-\operatorname{erf}\left(f\left(-N_{L} / 2\right)\right)\right] \\
& f(x)=\frac{\sqrt{-i}}{2}\left[\sqrt{\zeta^{\prime}\left(\Delta k_{0}+\delta k\right)} x l_{0}+\frac{\delta k}{\sqrt{\zeta^{\prime}\left(\Delta k_{0}+\delta k\right)}}\right] .
\end{aligned}
$$

The error function erf is defined as $\operatorname{erf}(x)=$ $2 / \sqrt{\pi} \int_{0}^{x} \exp \left(-y^{2}\right) d y$. Detailed inspection of the formula in Eq. (19) reveals that the larger the value of chirping parameter $\zeta^{\prime}$ the broader the phase-matching function $F^{\text {chirp }}(\Delta k)$.

As an example, we consider spectrally degenerate (nearly) collinear down-conversion from periodicallypoled $\mathrm{LiNbO}_{3}$ pumped at the wavelength $\lambda_{p}^{0}=775 \mathrm{~nm}$ by a cw laser beam. The signal and idler photons thus occur at the fiber-optics communication wavelength $\lambda_{s}=\lambda_{i}=1.55 \mu \mathrm{m}$. The crystal optical axis is perpendicular to the fields' propagation direction and is parallel to the vertical direction. All fields are vertically polarized and so the largest element $\chi_{33}^{(2)}$ of the susceptibility tensor is used. The natural phase mismatch for this configuration is compensated by the basic domain length $l_{0}$ equal to $9.51535 \mu \mathrm{m}$. A structure composed of $N_{L}=700$ layers is roughly $6.5 \mathrm{~mm}$ long and typically delivers $4 \times 10^{6}$ photon pairs per $100 \mathrm{~mW}$ of pumping in case of weakly random positions of boundaries (small values of variance $\sigma)$.

The most striking feature of RPSs is that the photonpair generation rate $N$ increases linearly with the number $N_{L}$ of domains, independently of the standard deviation $\sigma$ of the random positions of boundaries [see Fig. 1(a)]. Standard deviation $\sigma$ plays the central role in the determination of spectral widths $\Delta S_{s}$ and $\Delta S_{i}$ of the signal and idler fields. The larger the value of deviation $\sigma$ the broader the signal- and idler-field spectra $S_{s}$ and $S_{i}$, as documented in Fig. 1b. This behavior is easily understandable because structures generated with larger values of $\sigma$ have statistically a broader spatial spectrum of the $\chi^{(2)}(z)$ modulation which gives more freedom for the fulfillment of quasi-phase-matching conditions. It holds that the broader the signal- and idler-field spectra $S_{s}$ and $S_{i}$ the smaller the photon-pair generation rate $N$ [compare Figs. 1(a) and (b)]. It reflects the fact that constructive interference of fields from different domains is enhanced in the area outside the central frequencies $\omega_{s}^{0}$ and $\omega_{i}^{0}$ whereas this interference is weaken in the area around the central frequencies.

The photon-pair generation rate $N$ increases roughly linearly with the number $N_{L}$ of domains also in the case of 'weakly-random' structures described by the averaged squared modulus $\left\langle\left|F^{\mathrm{w}-\mathrm{r}}(\Delta k)\right|^{2}\right\rangle_{\text {av }}$ of phase-
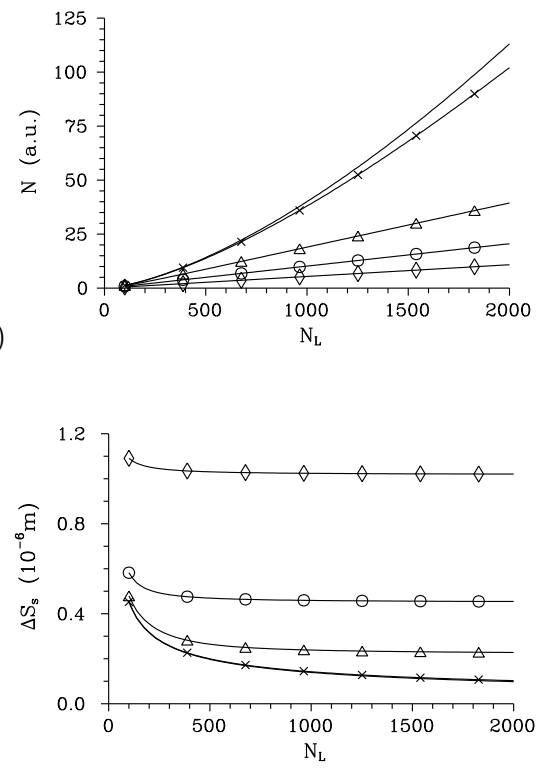

FIG. 1. (a) Photon-pair generation rate $N$ and (b) signal-field spectral width $\Delta S_{s}$ (FWHM) as functions of the number $N_{L}$ of domains for an ensemble of RPSs with standard deviation $\sigma$ equal to $0 \mathrm{~m}$ (solid curve), $0.1 \times 10^{-6} \mathrm{~m}$ (solid curve with $\times$ ), $0.5 \times 10^{-6} \mathrm{~m}$ (solid curve with $\triangle$ ), $1 \times 10^{-6} \mathrm{~m}$ (solid curve with $\circ$ ), and $2 \times 10^{-6} \mathrm{~m}$ (solid curve with $\left.\diamond\right)$; a.u. stands for arbitrary units.

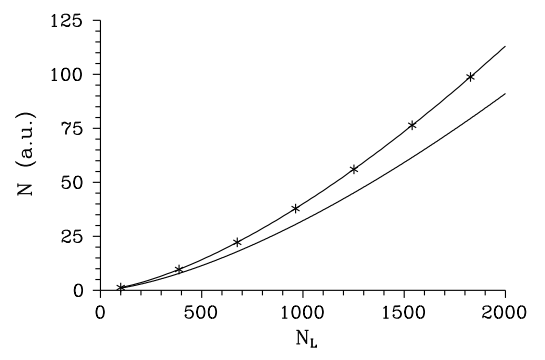

FIG. 2. Photon-pair generation rate $N$ as it depends on the number $N_{L}$ of domains for an ensemble of 'weakly-random' structures with standard deviation $\sigma$ equal to $0 \mathrm{~m}$ (solid curve with $*$ ) and $2 \times 10^{-6} \mathrm{~m}$ (solid curve).

matching function in Eq. (18), as shown in Fig. 2, The greater the standard deviation $\sigma$ the smaller the photonpair generation rate $N$. As for the signal-field spectral width $\Delta S_{s}$ its values do not practically depend on the variance $\sigma$.

The behavior of photon-pair generation as observed in RPSs can also be found in ordered CPPSs. Also here photon-pair generation rate $N$ is linearly proportional to the number $N_{L}$ of domains and spectral widths $\Delta S_{s}$ and $\Delta S_{i}$ increase with increasing chirping parameter $\zeta$. The main result of our analysis is that this similarity is both qualitative and quantitative. For any value of the chirping parameter $\zeta$ there exists a value of the standard deviation $\sigma$ such that spectral widths $\Delta S_{s}$ and $S \Delta_{i}$ of the generated signal and idler fields are the same. More- 
over (and a bit surprisingly), also photon-pair generation rates $N$ are comparable. This is illustrated in Fig. 3 for a chirped structure with $N_{L}=700$ domains. Its signalfield spectrum $S_{s}$ is extraordinarily broad (larger that $1 \mu \mathrm{m}$ ) for sufficiently large values of the chirping parameter $\zeta$ [see Fig. 3(a)]. Signal-field spectra $S_{s}$ of the same width can also be generated from RPSs with sufficiently large randomness (i.e., having large values of the deviation $\sigma$ ). Values of the standard deviation $\sigma$ corresponding to the values of chirping parameter $\zeta$ are plotted in Fig. 3(b). Photon-pair generation rates $N$ for RPSs and CPPSs are drawn for comparison in Fig. 3(c) in this case. Whereas CPPSs give better photon-pair generation rates $N$ for larger values of chirping parameter $\zeta$, RPSs even slightly overcome on average CPPSs for smaller values of $\zeta$. Moreover, the signal-field spectra $S_{s}$ of individual realizations may even be broader which results in sharper temporal features. On the other hand, these spectra are typically composed of many local peaks (see Fig. 4). RPSs thus represent an alternative broadband and efficient source of photon pairs with properties comparable to CPPSs. We note, that histograms of domain lengths corresponding to RPSs are broader compared to those characterizing CPPSs.

Alternatively, RPSs and CPPSs can be compared under the requirement of equal photon-pair generation rates $N$. Values of the photon-pair generation rate $N$ decrease with the increasing values of chirping parameter $\zeta$ [see Fig. 3(c)]. Transformation curve between standard deviation $\sigma$ and chirping parameter $\zeta$ stemming from the requirement of equal generation rates $N$ is monotonous and is plotted in Fig. 5 (a) in the considered case. The corresponding signal-field widths $\Delta S_{s}$ plotted in Fig. 囵(b) reveal that CPPSs provide broader spectra for the most of values of chirping parameter $\zeta$. However, the difference in spectral widths in CPPSs and RPSs is not dramatic.

The above presented results for RPSs represent an ensemble average. In practical applications, properties of individual realizations of a given RPS are naturally important. In Fig. 6, we show generation rates $N$ and signal-field widths $\Delta S_{s}$ for 10000 realizations of the RPS. Histograms of the generation rates $N$ and signal-field widths $\Delta S_{s}$ plotted in Figs. 6(b) and 6(c) are close to Gaussian distributions, in accordance with the central limiting theorem.

It holds that the larger the deviation $\sigma$ the smaller the relative quadratic fluctuations $\delta N$ and $\delta \Delta S_{s}$ of photonpair generation rates $N$ and signal-field spectral widths $\Delta S_{s}$, respectively $\left[\delta x=\sqrt{\left\langle(\Delta x)^{2}\right\rangle_{\mathrm{av}}} /\langle x\rangle_{\mathrm{av}}, \Delta x=x-\right.$ $\left.\langle x\rangle_{\text {av }}\right]$ (see Fig. [7). However, we should note that these relative fluctuations are quite large and can even approach $40 \%$.

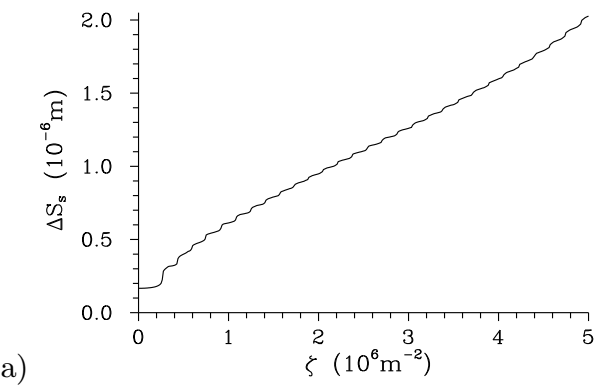

(b)
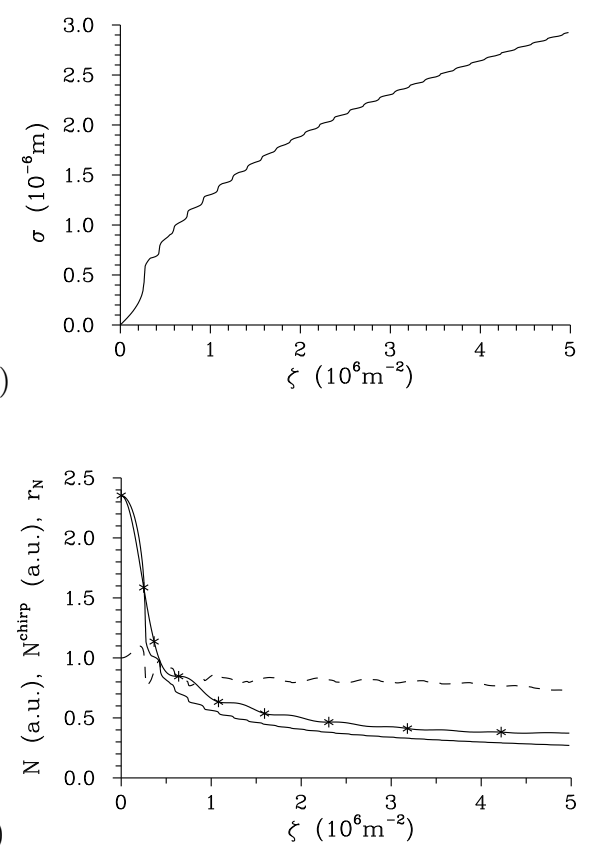

FIG. 3. (a) Signal-field spectral width $\Delta S_{s}$ (FWHM) as a function of chirping parameter $\zeta$. (b) Transformation curve between the standard deviation $\sigma$ and chirping parameter $\zeta$ assuming the same spectral widths $\Delta S_{s}$. (c) Photonpair generation rate for chirped ( $N^{\text {chirp }}$, solid curve with $*$ ) and random $\left(N\right.$, solid curve) structures and their ratio $r_{N}$ $\left(r_{N}=N / N^{\text {chirp }}\right.$, dashed curve $)$ as functions of chirping parameter $\zeta ; N_{L}=700$.

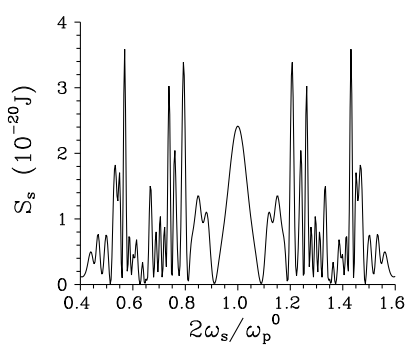

(a)

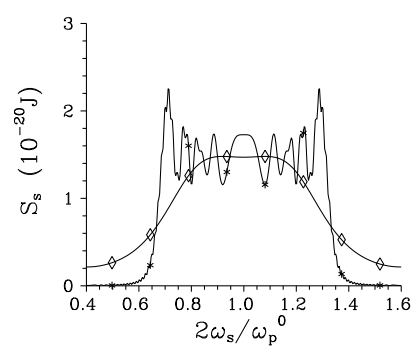

(b)
FIG. 4. Signal-field spectrum $S_{s}$ for (a) one typical realization of RPS (solid curve) and (b) CPPS (solid curve with $*$ ) and an ensemble of RPSs (solid curve with $\diamond$ ). Spectra $S_{s}$ are normalized such that one photon is emitted; $\sigma=2.1 \times 10^{-6} \mathrm{~m}$, $\zeta=2.5 \times 10^{6} \mathrm{~m}^{-2}, N_{L}=700$. 
(a)

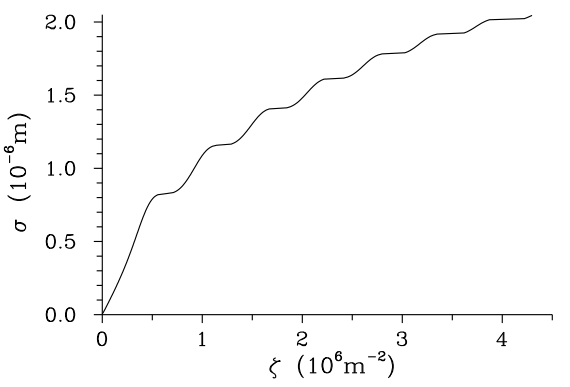

(b)

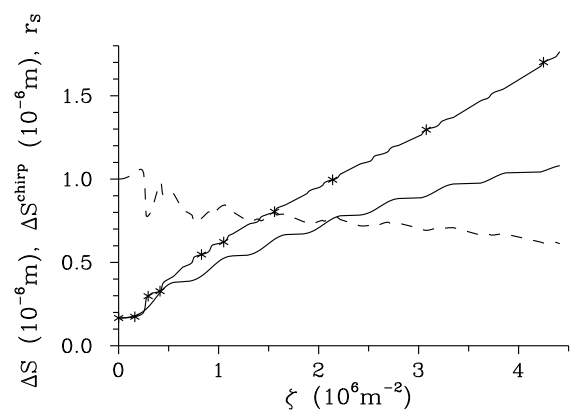

FIG. 5. (a) Transformation curve between the standard deviation $\sigma$ of RPSs and chirping parameter $\zeta$ assuming the same photon-pair generation rates $N$. (b) Signal-field spectral widths (FWHM) for random ( $\Delta S_{s}$, solid curve) and chirped $\left(\Delta S_{s}^{\text {chirp }}\right.$, solid curve with $\left.*\right)$ structures and their ratio $r_{S}$ $\left(r_{S}=S_{s} / S_{s}^{\text {chirp }}\right.$, dashed curve) as functions of chirping parameter $\zeta ; N_{L}=700$.

\section{TEMPORAL CORRELATIONS, ENTANGLEMENT TIME}

There occurs a strong correlation between possible detection times of a signal photon and its twin from one photon pair because both photons are generated inside the nonlinear medium at one instant. A finite distance between the detection times of both photons is a consequence of dispersion properties of the nonlinear medium through which both photons at different frequencies propagate before they leave the crystal. Temporal correlations of the signal and idler photons can be conveniently described using a two-photon temporal amplitude $\mathcal{A}$ defined as:

$$
\mathcal{A}\left(t_{s}, t_{i}\right)=\left\langle\operatorname{vac}\left|\hat{E}_{s}^{(+)}\left(t_{s}\right) \hat{E}_{i}^{(+)}\left(t_{i}\right)\right| \psi\right\rangle .
$$

This amplitude $\mathcal{A}\left(t_{s}, t_{i}\right)$ gives the probability amplitude of detecting a signal photon at time $t_{s}$ and an idler photon at time $t_{i}$.

The simplest experimental method for the determination of a typical constant characterizing temporal width of the two-photon detection window (entanglement time) uses a Hong-Ou-Mandel interferometer. In this interferometer, the signal and idler fields mutually interfere on a beam-splitter and photons leaving the beam-splitter at different output ports are subsequently detected in a coincidence-count measurement. The coincidence-count rate $R_{n}$ depends on a mutual time delay $\tau$ introduced

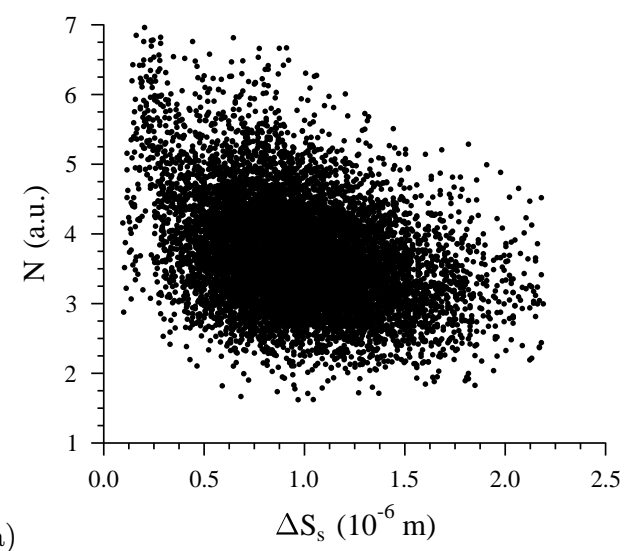

(b)
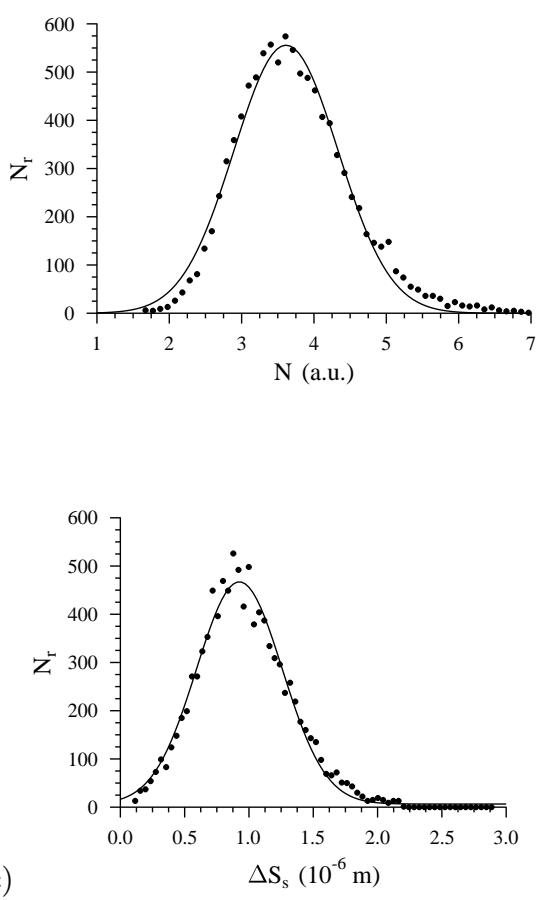

FIG. 6. (a) Photon-pair generation rates $N$ and signal-field spectral widths $\Delta S_{s}$ for 10000 realizations of RPSs (each realization is depicted as a point). (b), (c) Histograms of rates $N$ (b) and widths $\Delta S_{s}$ (c); $N_{r}$ gives the number of samples with given properties. Solid curves in (b) and (c) are best-fit Gaussian curves; $\sigma=2.1 \times 10^{-6} \mathrm{~m}, N_{L}=700$.

between the signal and idler photons. It can be shown that temporal extension of the interference part in the coincidence-count rate $R_{n}$ is proportional to entanglement time under certain conditions. The coincidencecount rate $R_{n}$ as a function of relative time delay $\tau$ is described by the following formula:

$$
R_{n}(\tau)=1-\varrho(\tau)
$$




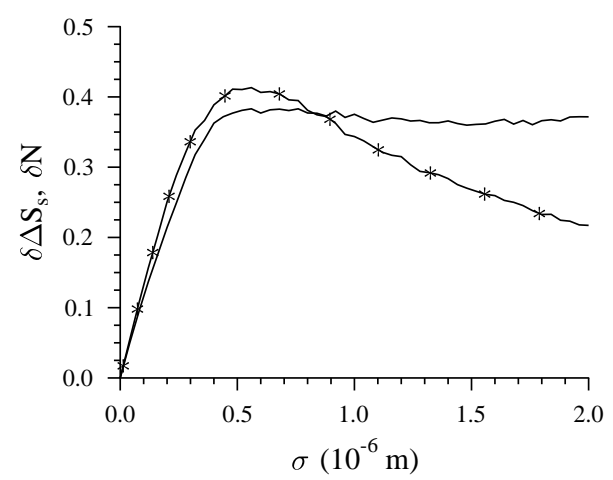

FIG. 7. Relative quadratic fluctuations $\delta \Delta S_{s}$ of the signalfield spectral width (solid curve) and relative quadratic fluctuations $\delta N$ of the photon-pair generation rate (solid curve with $*)$ as they depend on standard deviation $\zeta ; N_{L}=700$.

where

$$
\begin{aligned}
\rho(\tau)= & \frac{1}{2 R_{0}} \int_{-\infty}^{\infty} d t_{1} \int_{-\infty}^{\infty} d t_{2} \\
& \operatorname{Re}\left[\left\langle\mathcal{A}\left(t_{1}, t_{2}-\tau\right) \mathcal{A}^{*}\left(t_{2}, t_{1}-\tau\right)\right\rangle_{\mathrm{av}}\right] \\
R_{0}= & \frac{1}{2} \int_{-\infty}^{\infty} d t_{1} \int_{-\infty}^{\infty} d t_{2}\left\langle\left|\mathcal{A}\left(t_{1}, t_{2}\right)\right|^{2}\right\rangle_{\mathrm{av}}
\end{aligned}
$$

Inserting Eqs. (20) and (4) for the two-photon temporal amplitude $\mathcal{A}$ and quantum state $|\psi\rangle$, respectively, into Eqs. (22) and (23) we arrive at the expressions:

$$
\begin{aligned}
& \rho(\tau)=\frac{\pi \hbar^{2}}{4 \epsilon_{0}^{2} c^{2} \mathcal{B}^{2}} \frac{1}{R_{0}} \operatorname{Re}\left[\int_{0}^{\infty} d \omega_{s} \int_{0}^{\infty} d \omega_{i} \omega_{s} \omega_{i}\right. \\
& \left.\left\langle\Phi\left(\omega_{s}, \omega_{i}\right) \Phi^{*}\left(\omega_{i}, \omega_{s}\right)\right\rangle_{\mathrm{av}} \exp \left(i \omega_{i} \tau\right) \exp \left(-i \omega_{s} \tau\right)\right], \\
& R_{0}=\frac{\pi \hbar^{2}}{4 \epsilon_{0}^{2} c^{2} \mathcal{B}^{2}} \int_{0}^{\infty} d \omega_{s} \int_{0}^{\infty} d \omega_{i} \omega_{s} \omega_{i}\left\langle\left|\Phi\left(\omega_{s}, \omega_{i}\right)\right|^{2}\right\rangle_{\mathrm{av}} .
\end{aligned}
$$

For simplicity, we further assume cw-pumping with amplitude $\xi_{p}$ at frequency $\omega_{p}^{0}$, i.e. $E_{p}^{(+)}\left(\omega_{p}\right)=\xi_{p} \delta\left(\omega_{p}-\right.$ $\left.\omega_{p}^{0}\right)$. Formulas in Eqs. (24) and (25) can be simplified in this case and recast into the following form:

$$
\begin{aligned}
& \rho(\tau)= \frac{\hbar^{2}}{8 \epsilon_{0}^{2} c^{2} \mathcal{B}^{2}} \frac{\left|\xi_{p}\right|^{2}}{R_{0}} \operatorname{Re}\left[\exp \left(i \omega_{p}^{0} \tau\right) \int_{0}^{\infty} d \omega_{s}\right. \\
& \omega_{s}\left(\omega_{p}^{0}-\omega_{s}\right)\left|g\left(\omega_{s}, \omega_{p}^{0}-\omega_{s}\right)\right|^{2} \exp \left(-2 i \omega_{s} \tau\right) \\
&\left.\times \mathcal{F}\left(\Delta k\left(\omega_{s}, \omega_{p}^{0}-\omega_{s}\right), \Delta k\left(\omega_{p}^{0}-\omega_{s}, \omega_{s}\right)\right)\right] \\
& R_{0}= \frac{\hbar^{2}\left|\xi_{p}\right|^{2}}{8 \epsilon_{0}^{2} c^{2} \mathcal{B}^{2}} \int_{0}^{\infty} d \omega_{s} \omega_{s}\left(\omega_{p}^{0}-\omega_{s}\right)\left|g\left(\omega_{s}, \omega_{p}^{0}-\omega_{s}\right)\right|^{2} \\
& \times\left|\mathcal{F}\left(\Delta k\left(\omega_{s}, \omega_{p}^{0}-\omega_{s}\right), \Delta k\left(\omega_{s}, \omega_{p}^{0}-\omega_{s}\right)\right)\right|^{2}
\end{aligned}
$$

$g_{0} \equiv g\left(\omega_{s}^{0}, \omega_{i}^{0}\right)$. Function $\mathcal{F}$ introduced in Eqs. (26) and (27) incorporates phase-matching conditions into the description of temporal properties of photon pairs and is defined according to the formula:

$$
\mathcal{F}\left(\Delta k, \Delta k^{\prime}\right)=\left\langle F(\Delta k) F^{*}\left(\Delta k^{\prime}\right)\right\rangle_{\mathrm{av}} ;
$$

phase-matching function $F$ has been introduced in Eq. (10).

Considering RPSs with fluctuations of boundaries described by a Gaussian distribution in Eq. (8) function $\mathcal{F}$ in Eq. (28) takes the form:

$$
\begin{aligned}
\mathcal{F}\left(\Delta k, \Delta k^{\prime}\right)= & \frac{4}{\Delta k \Delta k^{\prime}} \tilde{\mathcal{F}}\left(\Delta k, \Delta k^{\prime}\right) \\
& \times \exp \left[-i\left(\Delta k-\Delta k^{\prime}\right) N_{L} l_{0}\right] \\
\tilde{\mathcal{F}}\left(\Delta k, \Delta k^{\prime}\right)= & \frac{1-H(D k)^{N_{L}+1}}{1-H(D k)}\left[\frac{H(\Delta k)}{H(\Delta k)+H(D k)}\right. \\
\times\left(-H(\Delta k) \frac{1-[-H(\Delta k)]^{N_{L}}}{1+H(\Delta k)}\right. & \\
& \left.-H(D k) \frac{1-[H(D k)]^{N_{L}}}{1-H(D k)}\right) \\
& \left.+\left(\Delta k \longleftrightarrow-\Delta k^{\prime}\right)\right]
\end{aligned}
$$

$D k=\Delta k-\Delta k^{\prime}$. Function $H$ occurring in Eq. (30) has been defined in Eq. (16). Symbol $\left(\Delta k \longleftrightarrow-\Delta k^{\prime}\right)$ in Eq. (30) replaces the term that is obtained by the indicated exchange applied to the preceded term inside the brackets.

Considering 'weakly-random' structures, the following 
form of the function $\tilde{\mathcal{F}}$ can be derived:

$$
\begin{gathered}
\tilde{\mathcal{F}}^{\mathrm{w}-\mathrm{r}}\left(\Delta k, \Delta k^{\prime}\right)=G(D k) \frac{1-\exp \left[i D k l_{0}\left(N_{L}+1\right)\right]}{1-\exp \left(i D k l_{0}\right)} \\
+\left[\frac { G ( \Delta k ) G ( \Delta k ^ { \prime } ) } { 1 - \operatorname { e x p } ( - i \Delta k ^ { \prime } l _ { 0 } ) } \left(\frac{1-\exp \left(i \Delta k N_{L} l_{0}\right)}{1-\exp \left(-i \Delta k l_{0}\right)}\right.\right. \\
\left.+\frac{1-\exp \left(-i D k N_{L} l_{0}\right)}{1-\exp \left(-i D k l_{0}\right)}\right) \\
\left.+\left(\Delta k \longleftrightarrow-\Delta k^{\prime}\right)\right] .
\end{gathered}
$$

On the other hand, function $\tilde{\mathcal{F}}^{\text {chirp }}$ attains a simple form in case of CPPSs:

$$
\tilde{\mathcal{F}}^{\text {chirp }}\left(\Delta k, \Delta k^{\prime}\right)=F^{\text {chirp }}(\Delta k) F^{\text {chirp } *}\left(\Delta k^{\prime}\right),
$$

where the formula for $F^{\text {chirp }}$ is written in Eq. (19).

Characteristics of temporal correlations (correlation time) between the signal and idler fields can also be obtained from the measurement of sum-frequency intensity in a nonlinear medium combining the signal and idler photons and having a sufficiently high value of $\chi^{(2) \text { sum }}$ nonlinearity. This process allows us to determine the temporal correlation function $I^{\text {sum }}$ of intensities of the signal and idler fields. Intensity $I^{\text {sum }}$ of the sumfrequency field is given along the formula:

$$
I^{\text {sum }}(\tau)=\eta^{\text {sum }} \int_{-\infty}^{\infty} d t\left|\left\langle\operatorname{vac}\left|\hat{E}_{s}^{(+)}(t) \hat{E}_{i}^{(+)}(t-\tau)\right| \psi\right\rangle\right|^{2}
$$

where constant $\eta^{\text {sum }}$ incorporates the value of $\chi^{(2) \text { sum }}$ nonlinearity and quantum detection efficiency.

The general formula in Eq. (33) can be recast into the following form using the expression for function $\mathcal{F}$ in Eq. (28):

$$
\begin{aligned}
& I^{\text {sum }}(\tau)=\frac{\eta^{\text {sum }} \hbar^{2}}{4 \varepsilon_{0}^{2} c^{2} \mathcal{B}^{2}} \int_{0}^{\infty} d \omega_{p}\left|E_{p}^{(+)}\left(\omega_{p}\right)\right|^{2} \\
& \quad \int_{0}^{\infty} d \omega_{s} \sqrt{\omega_{s}\left(\omega_{p}-\omega_{s}\right)} \int_{0}^{\infty} d \omega_{s}^{\prime} \sqrt{\omega_{s}^{\prime}\left(\omega_{p}-\omega_{s}^{\prime}\right)} \\
& \quad \times g\left(\omega_{s}, \omega_{p}-\omega_{s}\right) g^{*}\left(\omega_{s}^{\prime}, \omega_{p}-\omega_{s}^{\prime}\right) \exp \left[-i\left(\omega_{s}-\omega_{s}^{\prime}\right) \tau\right] \\
& \quad \times \mathcal{F}\left(\Delta k\left(\omega_{s}, \omega_{p}-\omega_{s}\right), \Delta k\left(\omega_{s}^{\prime}, \omega_{p}-\omega_{s}^{\prime}\right)\right) .
\end{aligned}
$$

When deriving Eqs. (33) and (34) we have assumed that the nonlinear medium in which sum-frequency generation occurs is ideally phase matched for frequencies present in the signal and idler fields.

A detailed analysis of the expression that gives the coincidence-count rate $R_{n}$ in a Hong-Ou-Mandel interferometer reveals an important property: the rate $R_{n}$ does not depend on phase variations along the signaland idler-field spectra in cw regime. This property is frequently referred as nonlocal dispersion cancellation [31, 32]. It follows that entanglement time $\Delta \tau^{\mathrm{HOM}}$ is inversely proportional to spectral widths $\Delta S_{s}$ and $\Delta S_{i}$

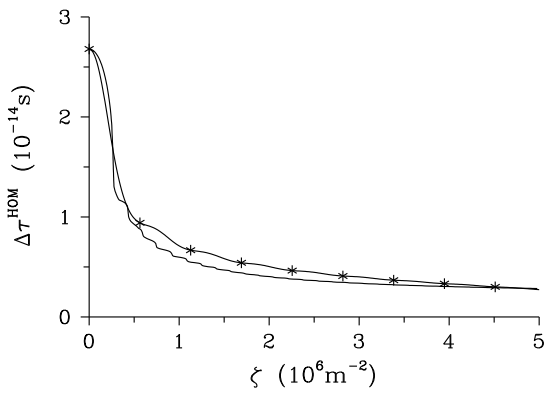

FIG. 8. Entanglement time $\Delta \tau^{\mathrm{HOM}}$ (FWHM) as a function of chirping parameter $\zeta$ for ensemble of RPSs with standard deviations $\sigma$ derived from the curve in Fig. 2b (solid curve) and CPPSs (solid curve with $*$ ); $N_{L}=700$.

of the signal and idler fields despite their complex profiles. We note that the entanglement time $\Delta \tau^{\mathrm{HOM}}$ is determined by a temporal extension (FWHM) of the coincidence-count interference pattern formed by the rate $R_{n}(\tau)$. Entanglement time $\Delta \tau^{\mathrm{HOM}}$ thus shortens with increasing values of the standard deviation $\sigma$ for RPSs. The dependence of entanglement time $\Delta \tau^{\mathrm{HOM}}$ on the deviation $\sigma$ for an ensemble of RPSs composed of 700 domains is shown in Fig. 8 , We can see in Fig. 8 that entanglement times $\Delta \tau^{\mathrm{HOM}}$ can be as short as several fs for sufficiently large values of the deviation $\sigma$. This indicates that temporal quantum correlations can be confined into an interval characterizing one optical cycle provided that spectral phase variations in the signal and idler fields are compensated. Entanglement times $\Delta \tau^{\mathrm{HOM}}$ of CPPSs with the same signal-field spectral widths $\Delta S_{s}$ are plotted in Fig. 8 for comparison that reveals nearly identical entanglement times of both types of structures. Typical coincidence-count interference patterns given by $R_{n}$ for photon pairs generated in both types of structures are compared in Fig. 9. They demonstrate a close similarity of photon-pairs behavior in a Hong-Ou-Mandel interferometer. There occur typical oscillations at the shoulders of the interference dips. Whereas regular oscillations characterize CPPSs, irregular oscillations with larger amplitudes occur for individual realizations of RPSs. However, widths of interference dips remain practically unchanged for different realizations of RPSs.

Correlation times $\Delta \tau^{\text {sum }}$ emerging from sum-frequency generation are in general longer than entanglement times $\Delta \tau^{\mathrm{HOM}}$ observed in a Hong-Ou-Mandel interferometer because of a strong phase modulation along the wide signal- and idler-field spectra $S_{s}$ and $S_{i}$ (see Fig. 10). Correlation times $\Delta \tau^{\mathrm{sum}}$ can be even an order of magnitude greater compared to entanglement times $\Delta \tau^{\mathrm{HOM}}$ for structures with ultra-broadband spectra. However, phase modulation along the spectrum can be compensated to certain extent which gives shorter correlation times $\Delta \tau^{\text {sum }}$. CPPSs have more regular spectral phase behavior (as demonstrated in Fig. 10) and quadratic phase compensation is usually sufficient to provide wavepackets several fs long. As for individual realizations of 


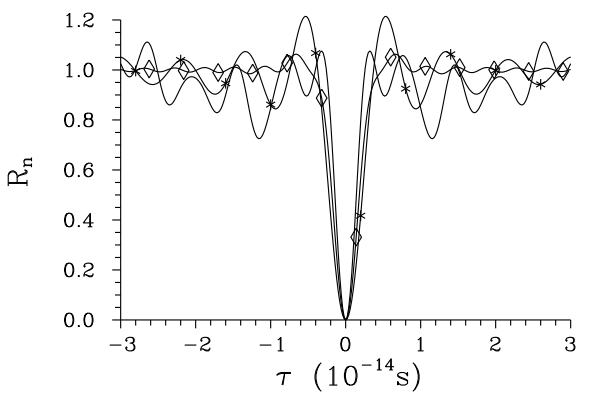

FIG. 9. Coincidence-count rate $R_{n}$ as it depends on relative time delay $\tau$ for one realization of RPS (solid curve), CPPS (solid curve with $*$ ), and an ensemble of RPSs (solid curve with $\diamond) ; \sigma=2.1 \times 10^{-6} \mathrm{~m}, \zeta=2.5 \times 10^{6} \mathrm{~m}^{-2}, N_{L}=700$.

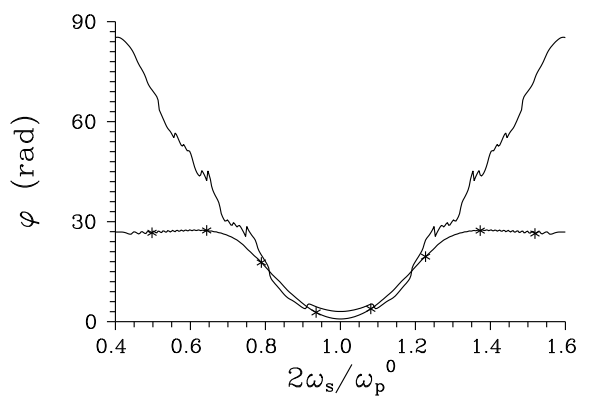

FIG. 10. Phase $\varphi$ of the two-photon spectral amplitude $\Phi\left(\omega_{s}, \omega_{p}^{0}-\omega_{s}\right)$ as it depends on normalized signal-field frequency $2 \omega_{s} / \omega_{p}^{0}$ for one realization of RPS (solid curve) and CPPS (solid curve with $*$ ); $\sigma=2.1 \times 10^{-6} \mathrm{~m}, \zeta=2.5 \times 10^{6} \mathrm{~m}^{-2}$, $N_{L}=700$.

RPSs, quadratic compensation is less efficient because of more irregular phase spectral behavior. Despite this values of temporal constants typical for chirped structures can be approached [see Fig. 11(a)]. Provided that an ideal phase compensation is reached, both types of structures give comparable results [see Fig. 11(b)] and are capable to generate photon pairs with wave-packets extending over the duration of one optical cycle. Experimentally, pulse shapers have been developed for this task and their capabilities in the area of photon pairs have already been demonstrated [33]. Comparison of the results obtained with quadratic and ideal compensations reveals that correlation times $\Delta \tau^{\text {sum }}$ are approx. two times larger if we restrict ourselves to quadratic compensation. Also the value of quadratic chirp that needs compensation differs for individual realizations of RPS. This requires an adaptive phase compensator. On the other hand phase compensation in case of CPPS can be reached in a simpler way, e.g., by inserting a peace of suitable material of defined length [34, 35]. Despite this RPSs are challenging both for basic physical experiments as well as metrology applications. (a)
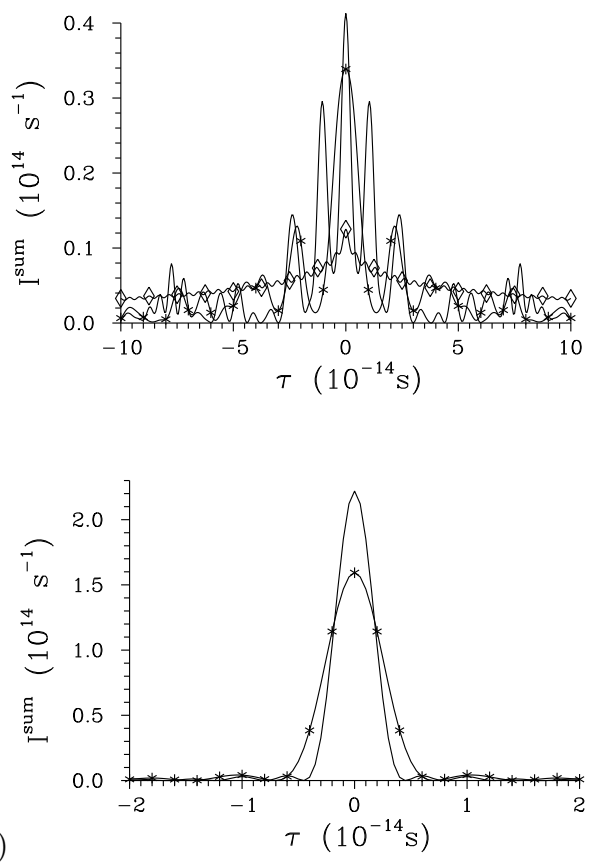

FIG. 11. Sum-frequency field intensity $I^{\text {sum }}$ as a function of relative time delay $\tau$ for one realization of RPS (solid curve), CPPS (solid curve with $*$ ), and an ensemble of RPSs (solid curve with $\diamond$ ). In (a) quadratic chirp in the signalfield amplitude spectrum is compensated for one realization of RPS and CPPS; in (b) complete spectral phase compensation is assumed. The curves are normalized such that $\int_{-\infty}^{\infty} d \tau I^{\operatorname{sum}}(\tau)=1 ; \sigma=2.1 \times 10^{-6} \mathrm{~m}, \zeta=2.5 \times 10^{6} \mathrm{~m}^{-2}$, $\bar{N}_{L}^{\infty}=700$.

\section{CORRELATIONS IN THE TRANSVERSE PLANE}

In order to describe spatial properties of the signal and idler beams (in the transverse plane) a simple generalization of the model presented in Sec. II has to be developed. The inclusion of phase-matching conditions also in the directions along the $x$ and $y$ axes and assumption of spectrally-flat transverse pump-beam profile $E_{p \perp}(x, y)$ result in the following separable form of a two-photon spectral amplitude $\Phi$ that additionally depends on radial $\left(\vartheta_{s}, \vartheta_{i}\right)$ and azimuthal $\left(\varphi_{s}, \varphi_{i}\right)$ signal- and idler-field emission angles (see Fig. 12):

$$
\begin{gathered}
\Phi\left(\omega_{s}, \omega_{i}, \vartheta_{s}, \varphi_{s}, \vartheta_{i}, \varphi_{i}\right)=\Phi_{z}\left(\omega_{s}, \omega_{i}, \vartheta_{s}, \varphi_{s}, \vartheta_{i}, \varphi_{i}\right) \\
\times \Phi_{x y}\left(\omega_{s}, \omega_{i}, \vartheta_{s}, \varphi_{s}, \vartheta_{i}, \varphi_{i}\right),
\end{gathered}
$$

where function $\Phi_{z}$ arises from phase-matching conditions in the $z$ direction and function $\Phi_{x y}$ originates in phase-matching conditions in the transverse $x y$ plane (see Fig. (11). Function $\Phi_{z}$ can be derived in analogy with the formula in Eq. (5):

$$
\begin{gathered}
\Phi_{z}\left(\omega_{s}, \omega_{i}, \vartheta_{s}, \varphi_{s}, \vartheta_{i}, \varphi_{i}\right)=g\left(\omega_{s}, \omega_{i}\right) E_{p}^{(+)}\left(\omega_{s}+\omega_{i}\right) \\
\times F\left(\Delta k\left(\omega_{s}, \omega_{i}, \vartheta_{s}, \varphi_{s}, \vartheta_{i}, \varphi_{i}\right)\right),
\end{gathered}
$$

where the stochastic function $F$ has been introduced in Eq. (6). Phase-matching conditions in the $x y$ plane give 


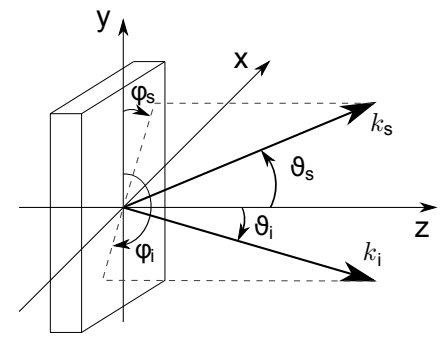

FIG. 12. Geometric scheme for the description of spatial properties. Direction of the signal- (idler-)field wave vector $k_{s}\left(k_{i}\right)$ is given by radial $\vartheta_{s}\left(\vartheta_{i}\right)$ and azimuthal $\varphi_{s}\left(\varphi_{i}\right)$ emission angles.

the function $\Phi_{x y}$ the following form:

$$
\begin{gathered}
\Phi_{x y}\left(\omega_{s}, \omega_{i}, \vartheta_{s}, \varphi_{s}, \vartheta_{i}, \varphi_{i}\right)=\int_{-\infty}^{\infty} d x \int_{-\infty}^{\infty} d y E_{p \perp}(x, y) \\
\times \exp \left(i \Delta k_{x} x+i \Delta k_{y} y\right)
\end{gathered}
$$

that includes a pump-beam amplitude profile $E_{p \perp}(x, y)$ in the transverse plane. Assuming normal incidence of the pump beam, the cartesian components of nonlinear phase mismatch in Eqs. (36) and (37) can be written as:

$$
\begin{aligned}
& \Delta k_{x}=k_{s}\left(\omega_{s}\right) \sin \left(\vartheta_{s}\right) \sin \left(\varphi_{s}\right)+k_{i}\left(\omega_{i}\right) \sin \left(\vartheta_{i}\right) \sin \left(\varphi_{i}\right) \\
& \Delta k_{y}=k_{s}\left(\omega_{s}\right) \sin \left(\vartheta_{s}\right) \cos \left(\varphi_{s}\right)+k_{i}\left(\omega_{i}\right) \sin \left(\vartheta_{i}\right) \cos \left(\varphi_{i}\right) \\
& \Delta k_{z}=k_{p}\left(\omega_{s}+\omega_{i}\right)-k_{s}\left(\omega_{s}\right) \cos \left(\vartheta_{s}\right)-k_{i}\left(\omega_{i}\right) \cos \left(\vartheta_{i}\right) .
\end{aligned}
$$

We assume a Gaussian pump-beam transverse profile in numerical calculations: $E_{p \perp}(x, y)=$ $1 /\left(\pi \Delta x_{p} \Delta y_{p}\right) \exp \left[-\left(x / \Delta x_{p}\right)^{2}-\left(y / \Delta y_{p}\right)^{2}\right]$ and $\Delta x_{p}$ $\left(\Delta y_{p}\right)$ stands for the pump-beam width along the $x(y)$ direction.

We first pay attention to transverse properties of the signal beam only. Its spectral density $s_{s}$ defined as

$$
\begin{aligned}
s_{s}\left(\omega_{s}, \vartheta_{s}, \varphi_{s}\right)= & \sin \left(\vartheta_{s}\right) \int d \omega_{i} \int d \vartheta_{i} \sin \left(\vartheta_{i}\right) \int d \varphi_{i} \\
& \left|\Phi\left(\omega_{s}, \omega_{i}, \vartheta_{s}, \varphi_{s}, \vartheta_{i}, \varphi_{i}\right)\right|^{2}
\end{aligned}
$$

depends on the signal-field radial $\left(\vartheta_{s}\right)$ and azimuthal $\left(\varphi_{s}\right)$ emission angles. As we study photon-pair emission near the collinear geometry, the signal beam (as well as the idler beam) has rotational symmetry along the $z$ axis. The dependence of spectral density $s_{s}$ on signal-field radial emission angle $\vartheta_{s}$ is shown in Fig. 13. Investigating one realization of RPS we observe a typical 'strip-like' behavior depicted in Fig. 13(a). Fixing the value of radial emission angle $\vartheta_{s}$ spectrum $s_{s}\left(\omega_{s}\right)$ is composed of many peaks occurring at positions specific for the studied realization [compare also with Fig. 4(a)]. Each peak changes continuously its central frequency as the radial emission angle $\vartheta_{s}$ moves. We note that this is typical also for layered structures that form band-gaps [6]. Averaging over many realizations of RPSs smoothes this 'strip-like' behavior [see Fig. 13(b)] and leads to that resembling
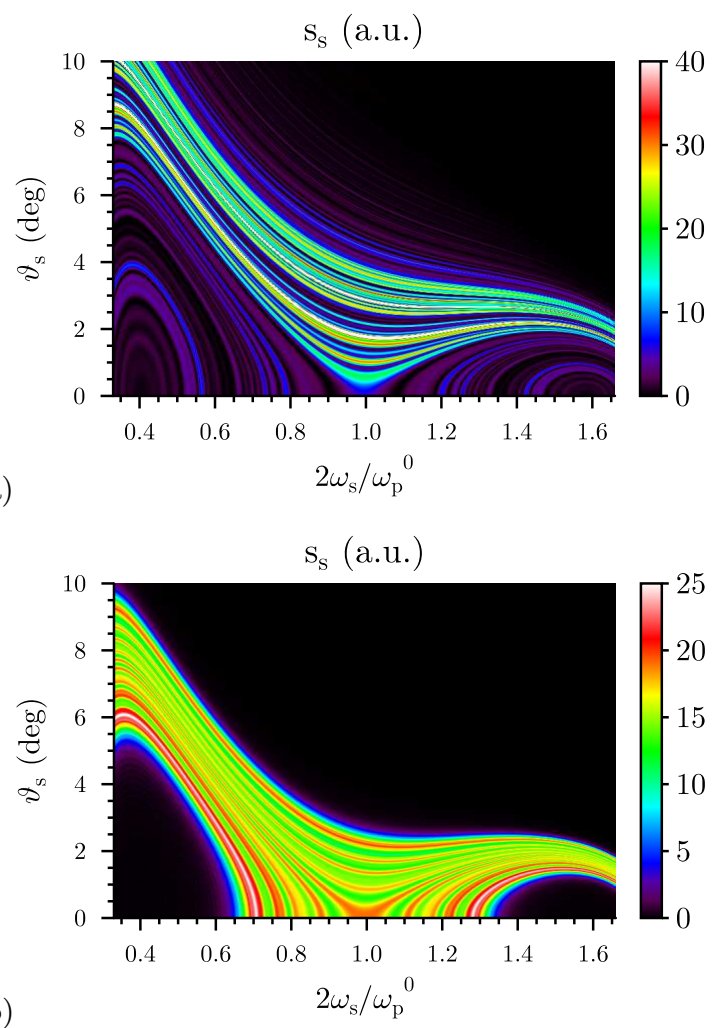

(b)

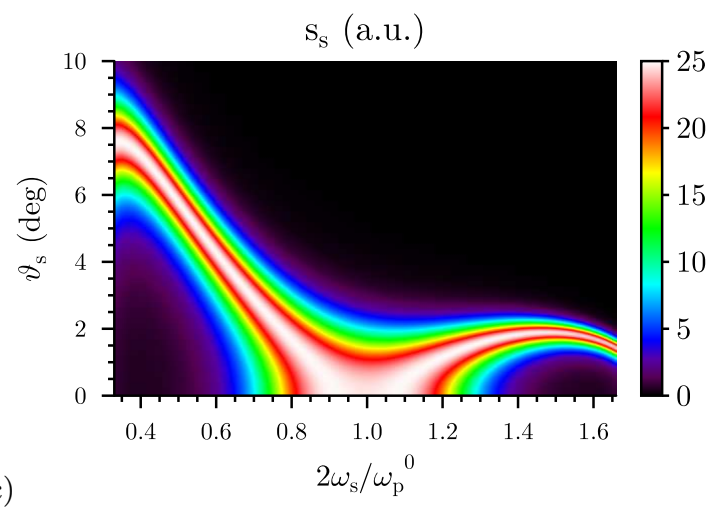

FIG. 13. Map of signal-field spectral density $s_{s}$ as it depends on signal-field radial emission angle $\vartheta_{s}$ for (a) one realization of RPS, (b) an ensemble of RPSs, and (c) CPPS; $\varphi_{s}=0 \mathrm{deg}$, $\sigma=2.1 \times 10^{-6} \mathrm{~m}, \zeta=2.5 \times 10^{6} \mathrm{~m}^{-2}, N_{L}=700$.

CPPSs [compare Figs. 13(b) and 13(c)]. In these cases spectral splitting is observed [36]. This behavior originates in phase-matching conditions along the $z$ direction.

Integration of spectral densities $s_{s}$ over the signal-field frequency $\omega_{s}$ gives us densities $n_{s}$ of photon-pair numbers that are plotted in Fig. 14 for the structures studied in Fig. 13. Whereas the profile of density $n_{s}\left(\vartheta_{s}\right)$ is complex for one realization of RPS, typical shapes with one maximum around a nonzero value of $\vartheta_{s}$ characterize the profiles of density $n_{s}\left(\vartheta_{s}\right)$ for an ensemble of RPSs and CPPS. 


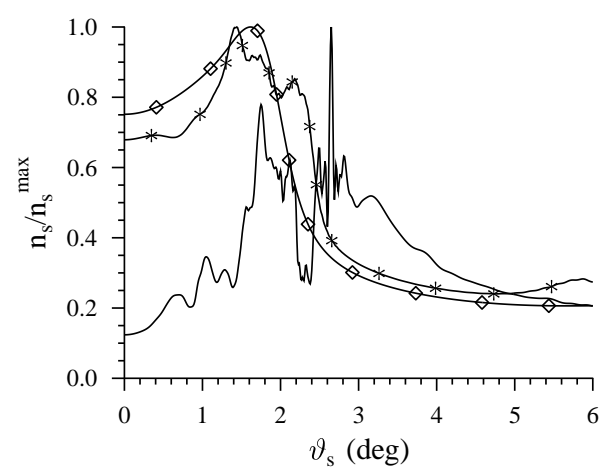

FIG. 14. Profile of density $n_{s}$ of signal-field photon numbers as a function of signal-field radial emission angle $\vartheta_{s}$ for one realization of RPS (solid curve), CPPS (solid curve with $*$ ), and an ensemble of RPSs (solid curve with $\diamond) ; n_{s}^{\max }=\max _{\vartheta_{s}}\left[n_{s}\left(\vartheta_{s}\right)\right]$. Plane-wave pumping is assumed; $n_{s}\left(\vartheta_{s}, \psi_{s}\right)=\int d \omega_{s} s_{s}\left(\omega_{s}, \vartheta_{s}, \varphi_{s}\right) ; \varphi_{s}=0 \mathrm{deg}, \sigma=$ $2.1 \times 10^{-6} \mathrm{~m}, \zeta=2.5 \times 10^{6} \mathrm{~m}^{-2}, N_{L}=700$.

Correlated area $g_{i}$ of an (idler) photon in a pair represents spatial analogy to entanglement time and characterizes correlations of photon twins in the transverse plane. By definition, it gives probability of emitting an idler photon into radial emission angle $\vartheta_{i}$ and azimuthal emission angle $\varphi_{i}$ provided that its signal twin has been emitted in a fixed radial emission angle $\vartheta_{s}$ and azimuthal emission angle $\varphi_{s}$, i.e.:

$$
\begin{aligned}
& g_{i}\left(\vartheta_{i}, \varphi_{i} ; \vartheta_{s}, \varphi_{s}\right)=\sin \left(\vartheta_{s}\right) \sin \left(\vartheta_{i}\right) \\
& \quad \times \int d \omega_{s} \int d \omega_{i}\left|\Phi\left(\omega_{s}, \omega_{i}, \vartheta_{s}, \varphi_{s}, \vartheta_{i}, \varphi_{i}\right)\right|^{2} .
\end{aligned}
$$

Because we mainly pay attention to beams propagating in the vicinity of the $z$ axis, we assume that the signal photon is emitted along the $z$ axis $\left(\vartheta_{s}=\varphi_{s}=\right.$ $0 \mathrm{deg}$ ). The correlated area as described by function $g_{i}$ in Eq. (40) then has rotational symmetry and its profiles along the radial emission angle $\vartheta_{i}$ for CPPS and an ensemble of RPSs nearly coincide, as documented in Fig. 15(a). On the other hand, broader profiles are typical for individual realizations of RPSs. These individual realizations form compact correlated areas without large local peaks (compare with Fig. 4 where spectrum $S_{s}$ for one realization of RPS is plotted). The width $\Delta \vartheta_{i}$ of the correlated area along the radial angle $\vartheta_{i}$ depends in general on phase-matching conditions along the $z$ and $\vartheta_{i}$ axes. Thus length of the structure, pump-field (temporal) spectral width as well as width of the pump-beam waist determine together the width $\Delta \vartheta_{i}$ (for more details, see [36]). For example, focusing the pump beam, values of the radial width $\Delta \vartheta_{i}$ can be varied nearly by one order of magnitude [see Fig. 15(b)]. This behavior can be easily explained by the fact that the more the pump beam is focused, the wider its spatial spectrum in the transverse plane, and so the weaker the phase-matching conditions in this plane.

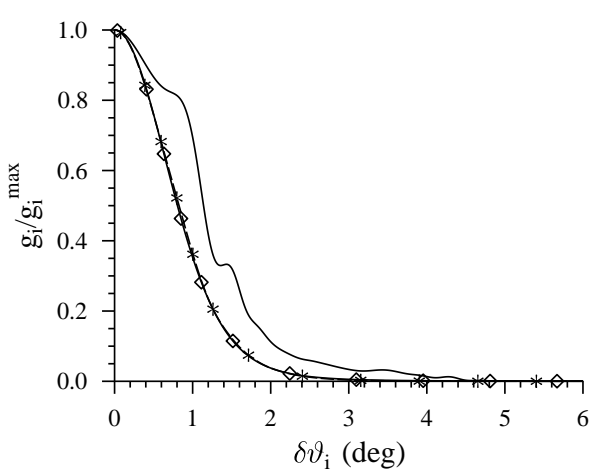

(a)

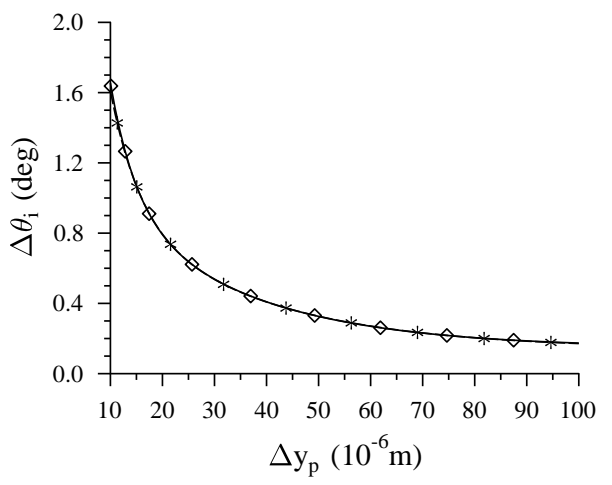

FIG. 15. (a) Radial profile $g_{i}\left(\vartheta_{i}\right)$ of the correlated area for $\Delta y_{p}=1 \times 10^{-5} \mathrm{~m}$ and (b) radial width $\Delta \vartheta_{i}$ of the correlated area as it depends on pump-beam width $\Delta y_{p}$ for one realization of RPS (solid curve), CPPS (solid curve with $*$ ), and an ensemble of RPSs (solid curve with $\diamond) ; \vartheta_{i}=\vartheta_{i}^{0}+\delta \vartheta_{i}$; $g_{i}^{\max }=\max _{\vartheta_{i}}\left[g_{i}\left(\vartheta_{i}\right)\right]$. Radially symmetric pump beam is assumed, i.e. $\Delta x_{p}=\Delta y_{p} ; \varphi_{s}=\vartheta_{s}=0 \mathrm{deg}, \varphi_{i}=180 \mathrm{deg}$, $\vartheta_{i}^{0}=0 \mathrm{deg} ; \sigma=2.1 \times 10^{-6} \mathrm{~m}, \zeta=2.5 \times 10^{6} \mathrm{~m}^{-2}, N_{L}=700$.

\section{THE ROLE OF TEMPERATURE}

We have seen that an ensemble of RPSs and a CPPS have similar properties. This close similarity is preserved also when studying temperature dependencies [21] that are in general weak. On the other hand, behavior of individual realizations of RPSs manifests a stronger temperature dependence. However, influence of temperature varies from realization to realization. Whereas properties of the realization of RPS studied above do not considerably change with temperature (see Fig. 16 for the signalfield spectral width $\Delta S_{s}$ in the temperature range from 284 to $300 \mathrm{~K}$ ), other realizations are more prone to the change of temperature. This can be conveniently used for efficient temperature modifications of properties of photon pairs. We note that these effects have their origin in temperature dependence of indexes of refraction [21]. 


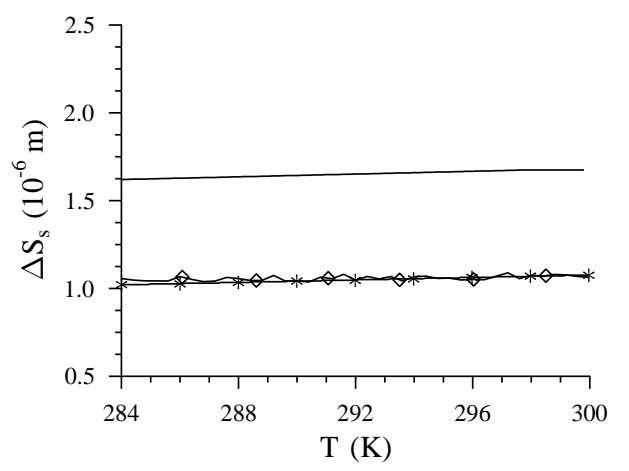

FIG. 16. Signal-field spectral width $\Delta S_{s}$ as it depends on temperature $T$ for one realization of RPS (solid curve), CPPS (solid curve with $*$ ), and an ensemble of RPSs (solid curve with $\diamond) ; \sigma=2.1 \times 10^{-6} \mathrm{~m}, \zeta=2.5 \times 10^{6} \mathrm{~m}^{-2}, N_{L}=700$.

\section{THE ROLE OF SMALL RANDOM (FABRICATION) ERRORS}

In the fabrication process, a small random error necessarily occurs [37]. This error is sometimes called a duty cycle error and, in general, leads to lowering of photon-pair emission rates [18]. Considering spectral widths, they are resistant against this error in uniformly periodically-poled crystals [37] [see also Eq. (18) valid for 'weakly-random' structures]. On the other hand, spectral widths are slightly reduced in CPPSs as documented in Fig. 17 We can see in Fig. 17 that a (large) fabrication error with variance $\sigma_{\mathrm{er}}=5 \times 10^{-7} \mathrm{~m}$ results in the reduction of signal-field spectral width $\Delta S_{s}$ only by approx. $10 \%$. Individual realizations of RPSs are much more sensitive to the fabrication error. The observed spectral changes depend on individual realizations. As an example, the signal-field spectral width $\Delta S_{s}$ of the sample analyzed above decreases with the increasing variance $\sigma_{\text {er }}$ of the fabrication error. This is natural, because spectrum of this realization is broader compared to the ensemble mean value. We note that it holds also here that the narrower the signal-field spectrum, the greater the photon-pair generation rate $N$ and vice versa.

\section{THE ROLE OF ORDERING IN CHIRPED PERIODICALLY-POLED STRUCTURES}

The benefit of ordering of individual domains by their lengths in CPPS can be quantified as follows. We take an ordered structure and divide it into segments containing $d$ domains. We then randomly position these segments in a new artificial structure and finally obtain mean values of physical quantities after averaging over random positions. In the limiting case of $d=1$ we have a completely random structure similar to those studied above. It can be shown that the signal-field spectral

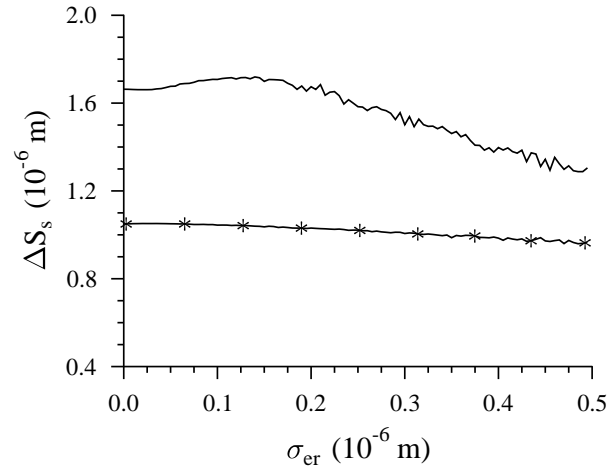

FIG. 17. Signal-field spectral width $\Delta S_{s}$ as a function of variance $\sigma_{\text {er }}$ of the fabrication error for one realization of RPS (solid curve) and CPPS (solid curve with $*$ ). Averaging over the fabrication error was done in 1000 randomly chosen positions; $\sigma=2.1 \times 10^{-6} \mathrm{~m}, \zeta=2.5 \times 10^{6} \mathrm{~m}^{-2}, N_{L}=700$.

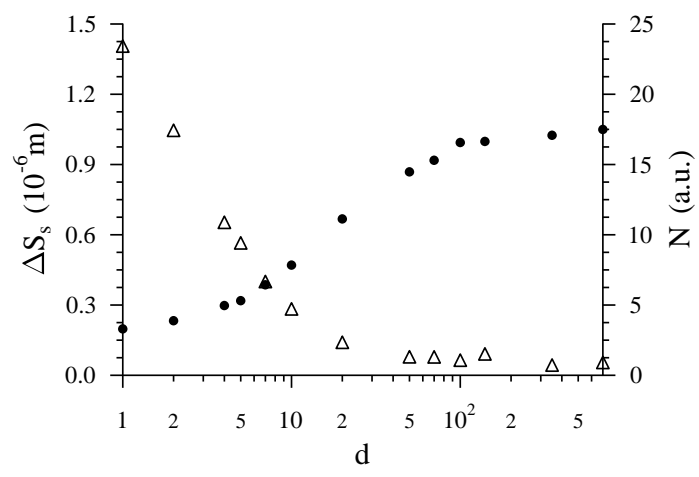

FIG. 18. Signal-field spectral width $\Delta S_{s}$ (solid curve with •) and photon-pair generation rate $N$ (solid curve with $\triangle$ ) as functions of segment length $d$. Averaging over 1000 random positions of segments was used in calculations. $\zeta=2.5 \times$ $10^{6} \mathrm{~m}^{-2}, N_{L}=700$.

width $\Delta S_{s}$ decreases with the decreasing segment length $d$ (see Fig. 18). This is accompanied by an increase of photon-pair generation rate $N$. This behavior reflects the fact that spectra of the fields coming from individual domains are combined in a more constructive way in the central spectral area with the increasing randomness (decreasing value of segment length $d$ ). The graph in Fig. 18 also demonstrates that the requirement for the same spectral widths $\Delta S_{s}$ of RPSs and CPPS inevitably implies that the histogram of domains' lengths for RPSs is broader than that obtained for CPPS.

\section{CONCLUSIONS}

Properties of photon pairs generated in randomly poled structures have been found quantitatively similar to those characterizing chirped periodically-poled structures. Es- 
pecially, ultra broadband signal and idler fields can be emitted in randomly poled structures. The accompanying sharp mutual temporal correlations of the signal and idler fields can even reach the duration of a single-photon cycle (several fs). Photon-pair generation rates depending linearly on the number of domains are specific to random structures. Stronger temperature dependencies of parameters characterizing photon pairs in random structures (their individual realizations) compared to those found in chirped periodically-poled crystals have been observed. In general, application potential of randomly poled structures similar to that of chirped periodicallypoled structures has been revealed. Contrary to chirped periodically-poled structures randomly poled structures do not require high precision in their fabrication. This is a great promise for the use of randomly poled structures.

\section{ACKNOWLEDGMENTS}

Support by projects IAA100100713 of GA AV ČR, COST OC 09026, 1M06002 of the Czech Ministry of Education and the Operational Program Research and Development for Innovations - European Social Fund (CZ.1.05/2.1.00/03.0058) are acknowledged. J. S. was supported by the project PrF-2010-009 of Palacký University.
[1] P. A. Franken, A. E. Hill, C. W. Peters, and G. Weinreich, Phys. Rev. Lett. 7, 118 (1961).

[2] R. Ghosh, C. K. Hong, Z. Y. Ou, and L. Mandel, Phys. Rev. A 34, 3962 (1986).

[3] M. H. Rubin, D. N. Klyshko, Y. H. Shih, and A. V. Sergienko, Phys. Rev. A 50, 5122 (1994).

[4] J. A. Armstrong, N. Bloembergen, J. Ducuing, and P. S. Pershan, Phys. Rev. 127, 1918 (1962).

[5] D. S. Hum and M. M. Fejer, Comptes Rendus Physique 8, 180 (2007).

[6] J. Peřina Jr., M. Centini, C. Sibilia, M. Bertolotti, and M. Scalora, Phys. Rev. A 73, 033823 (2006).

[7] M. Centini, J. Peřina Jr., L. Sciscione, C. Sibilia, M. Scalora, M. J. Bloemer, and M. Bertolotti, Phys. Rev. A 72, 033806 (2005).

[8] A. N. Vamivakas, B. E. A. Saleh, A. V. Sergienko, and M. C. Teich, Phys. Rev. A 70, 043810 (2004).

[9] J. Peřina Jr., M. Centini, C. Sibilia, M. Bertolotti, and M. Scalora, Phys. Rev. A 75, 013805 (2007).

[10] J. Peřina Jr., A. Lukš, O. Haderka, and M. Scalora, Phys. Rev. Lett. 103, 063902 (2009).

[11] J. Peřina Jr., A. Lukš, and O. Haderka, Phys. Rev. A 80, 043837 (2009).

[12] M. Baudrier-Raybaut, R. Haidar, P. Kupecek, P. Lemasson, and E. Rosencher, Nature 432, 374 (2004).

[13] E. Y. Morozov and A. S. Chirkin, Journal of Russian Laser Research 25, 013902 (2004).

[14] X. Vidal and J. Martorell, Phys. Rev. Lett. 97, 013902 (2006).

[15] A. S. Aleksandrovsky, A. M. Vyunishev, I. E. Shakhura, A. I. Zaitsev, and A. V. Zamkov, Phys. Rev. A 78, 031802 (2008).

[16] M. Centini, D. Felbacq, D. S. Wiersma, C. Sibilia, M. Scalora, and M. Bertolotti, J. Eur. Opt. Soc. Rapid Publ. 1, 06021 (2006).

[17] G. K. Kitaeva, Phys. Rev. A 76, 043841 (2007).

[18] J. S. Pelc, C. Langrock, Q. Zhang, and M. M. Fejer, Opt. Lett. 35, 2804 (2010).
[19] R. Fischer, S. M. Saltiel, D. N. Neshev, W. Krolikowski, and Y. S. Kivshar, Central European Journal of Physics 6, 569 (2008).

[20] J. Svozilík and J. Peřina Jr., Opt. Express 18, 27130 (2010).

[21] M. B. Nasr, S. Carrasco, B. E. A. Saleh, A. V. Sergienko, M. C. Teich, J. P. Torres, L. Torner, D. S. Hum, and M. M. Fejer, Phys. Rev. Lett. 100, 183601 (2008).

[22] S. E. Harris, Phys. Rev. Lett. 98, 063602 (2007).

[23] J. Svozilík and J. Peřina Jr., Phys. Rev. A 80, 023819 (2009).

[24] M. F. Saleh, B. E. A. Saleh, and M. C. Teich, Phys. Rev. A 79, 053842 (2009).

[25] S. Carrasco, J. P. Torres, L. Torner, A. V. Sergienko, B. E. A. Saleh, and M. C. Teich, Opt. Lett. 29, 2429 (2004).

[26] T. S. Humble and W. P. Grice, Phys. Rev. A 75, 022307 (2007).

[27] P. P. Rohde, T. C. Ralph, and M. A. Nielsen, Phys. Rev. A 72, 052332 (2005).

[28] K. A. O'Donnell and A. B. U'Ren, Opt. lett. 32, 817 (2007).

[29] C. K. Hong and L. Mandel, Phys. Rev. A 31, 2409 (1985).

[30] B. E. A. Saleh and M. C. Teich, Fundamentals of Photonics (Wiley, New York, 1991).

[31] A. M. Steinberg, P. G. Kwiat, and R. Y. Chiao, Phys. Rev. Lett. 68, 2421 (1992).

[32] J. Peřina Jr., A. V. Sergienko, B. M. Jost, B. E. A. Saleh, and M. C. Teich, Phys. Rev. A 59, 2359 (1999).

[33] B. Dayan, Y. Bromberg, I. Afek, and Y. Silberberg, Phys. Rev. A 75, 043804 (2007).

[34] G. Brida, M. V. Chekhova, I. P. Degiovanni, M. Genovese, G. K. Kitaeva, A. Meda, and O. A. Shumilkina, Phys. Rev. Lett. 103, 193602.

[35] S. Sensarn, G. Y. Yin, and S. E. Harris, Phys. Rev. Lett. 104, 253602 (2010).

[36] M. Hamar, J. Peřina Jr., O. Haderka, and V. Michálek, Phys. Rev. A 81, 043827 (2010).

[37] M. M. Fejer, G. A. Magel, D. H. Jundt, and R. L. Byer, IEEE J. Quant. Electr. 28, 2631 (1992). 